A N N A L E S Annales de Bretagne et des Pays de l'Ouest

\title{
L'amnistie de 1859 et le retour des mariannistes en Anjou : entre pardon judiciaire et surveillance policière
}

\section{Boris Battais}

\section{(2) OpenEdition \\ Journals}

Édition électronique

URL : http://journals.openedition.org/abpo/1770

DOI : 10.4000/abpo. 1770

ISBN : 978-2-7535-1518-5

ISSN : 2108-6443

Éditeur

Presses universitaires de Rennes

Édition imprimée

Date de publication : 10 juillet 2010

Pagination : 69-83

ISBN : 978-2-7535-1214-6

ISSN : 0399-0826

\section{Référence électronique}

Boris Battais, «L'amnistie de 1859 et le retour des mariannistes en Anjou : entre pardon judiciaire et surveillance policière ", Annales de Bretagne et des Pays de l'Ouest [En ligne], 117-2 | 2010, mis en ligne le 20 juillet 2012, consulté le 21 avril 2019. URL : http://journals.openedition.org/abpo/1770 ; DOI : $10.4000 / a b p o .1770$ 


\title{
L'amnistie de 1859 et le retour des mariannistes en Anjou : entre pardon judiciaire et surveillance policière
}

\author{
Boris BATTAIS \\ Doctorant, \\ CEHRIO - université d'Angers
}

Dans la nuit du 26 au 27 août 1855, plusieurs centaines d'ouvriers, majoritairement des ardoisiers trélazéens, marchent sur Angers, chef-lieu du Maine-et-Loire. Pour la plupart affiliés à la société secrète républicaine la Marianne, ils réclament la baisse du prix du pain et veulent renverser le régime impérial pour restaurer la république démocratique et sociale. Attendus par la troupe au niveau de la rue Bressigny à Angers, les insurgés sont dispersés et plus d'une centaine d'entre eux sont arrêtés sans qu'un coup de feu n'ait, semble-t-il, été tiré. D'autres arrestations ont lieu dans tout le département durant les semaines suivantes.

Comme le montre Jacques-Guy Petit, l'insurrection, comme son traitement judiciaire, répond à des situations politiques qui dépassent le simple cadre local $^{1}$. L'existence de la société secrète, bien antérieure à 1855 , est avérée dans toute la vallée de la Loire. Constituant une réponse à la politique de répression du Parti de l'Ordre depuis 1849, la Marianne angevine n'est, à cette date, ni isolée ni récente. En 1853, et après de longues hésitations, les enquêtes menées de Paris dans toute la France permettent aux autorités de mettre en relation la société angevine avec celle du même nom dans les départements du $\mathrm{Cher}^{2}$, de la Nièvre et de l'Allier. On est aussi désormais certain de l'existence de deux réseaux essentiels qui fusionnent cette même année sur la demande de Ledru-Rollin, alors exilé à Londres,

1. Petrt, Jacques-Guy, " Marianne en Anjou : l'insurrection des ardoisiers de Trélazé ", Annales de Bretagne et des pays de l'Ouest, 104, 3, 1997, p. 188-200.

2. Sur le mouvement ouvrier dans le département du Cher, voir Pigenet, Michel, Les Ouvriers du Cher (fin XVII e siècle-1914) : travail, espace et conscience sociale, Paris, Institut CGT d'Histoire Sociale, 1990, p. 179. 
la Jeune montagne, à Paris, et dans le Val de Loire la Marianne. Dès lors, les nombreux procès qui ont eu lieu à Paris, Tours ou Angers témoignent de la volonté manifeste des autorités impériales de les démanteler.

En 1855, dans le contexte difficile de la guerre de Crimée et après l'attentat de Pianori, des rumeurs laissent entendre que l'Empereur serait mort et que le retour à la République serait imminent ${ }^{3}$. Des bruits d'insurrection générale pour la nuit du 23 au 24 février circulent même dans le Cher et la Nièvre. Jean-Marie Secrétain, le chef politique des perrayeux angevins et trélazéens, se rend du 18 au 25 août à Paris, sans doute pour aller chercher le mot d'ordre d'insurrection. Il revient persuadé qu'un mouvement républicain national et simultané doit avoir lieu.

S'il se singularise par l'ampleur de la répression et la sévérité des condamnations prononcées, le traitement judiciaire de l'affaire révèle l'acharnement du régime impérial pour se débarrasser de l'opposition républicaine, ainsi qu'un réel souci d'exemplarité à dimension nationale ${ }^{4}$ : les mariannistes, Secrétain en tête, sont en fait manipulés au profit de l'Empire qui veut faire de l'éradication de la Marianne un exemple dans la guerre qu'il a déclarée aux sociétés secrètes. Aussi, les suites immédiates de l'affaire traduisent-elles les orientations et la nature du régime. Les mariannistes sont les victimes certes, mais aussi les instruments parmi d'autres, de la politique répressive de l'Empire autoritaire. Mais pour qui repousse les horizons de l'affaire, le décret impérial d'amnistie du 16 août 1859 pour les condamnés politiques depuis 1848 doit être lui aussi appréhendé dans un contexte national et international particulier, les choix politiques intérieurs et extérieurs de Napoléon III à la fin de la première décennie de son règne conditionnant son action à l'égard des opposants politiques et l'obligeant à se trouver de nouveaux appuis dans la société d'alors.

En quoi le décret impérial d'amnistie de 1859 constitue-t-il une décision révélatrice à la fois de la nature du régime, de son fonctionnement, de ses hésitations et finalement de ses évolutions libérales? En quoi cette amnistie et le retour des mariannistes en Anjou entre 1859 et 1860, comme celui de tous les condamnés depuis 1848 d'ailleurs, peuvent-ils dès lors apparaître comme une instrumentalisation politique de l'État impérial à ses propres fins? Comment, enfin, les autorités impériales, locales et nationales, entendent-elles contrôler et réguler les effets politiques et sociaux du retour de ces " libérés de 1859 " qui, jadis jugés " incorrigibles ", demeurent des " dangers potentiels " pour l'ordre impérial napoléonien?

3. Sur les rumeurs, voir les travaux de François Ploux, notamment « Imaginaire politique et représentations sociales : les rumeurs dans les campagnes françaises sous le Second Empire ", Ruralia, 1999-05, [en ligne], mis en ligne le 25 janvier 2005. [http:// ruralia.revues.org/document109.html]; et De bouche à oreille : naissance et propagation des rumeurs dans la France du XIX ${ }^{e}$ siècle Paris, Aubier, 2003, 289 p.

4. Plus généralement sur les rapports entre l'État et les sociétés secrètes, voir PRICE, Roger, The French Second Empire : an anatomy of political power, Cambridge, Cambridge University Press, 2001, 507 p. 


\section{" Empire autoritaire " ou " empire libéral " : les mariannistes au cœur des dilemmes politiques du régime impérial}

Depuis 1852 et la mise en place de la dictature bonapartiste, le pouvoir, appuyé par le parti de l'Ordre, poursuit la politique très répressive et autoritaire initiée en 1849. C'est la période de ce qu'il est convenu d'appeler " l'Empire autoritaire ». Cependant, à partir de 1858, des événements suscitent la remise en cause des bases politiques et sociales du régime et obligent l'Empire à se chercher de nouveaux appuis.

\section{L'attentat d'Orsini et l'apogée de la politique répressive impériale}

Le soir du 14 janvier 1858 à Paris, le couple impérial se rend à l'Opéra. Au moment où le couple s'apprête à entrer dans le théâtre, trois bombes sont lancées. L'Empereur et l'Impératrice sont indemnes mais huit personnes sont tuées et plus de 150 sont blessées. Très vite, quatre Italiens sont arrêtés, dont leur chef, Orsini, un ancien membre de la République romaine. Il ne cache pas sa culpabilité. Pour lui, Napoléon III mort, la république serait inévitable en France et celle-ci aiderait l'Italie à se libérer ${ }^{5}$. Finalement, bien que défendu par J. Favre, il est condamné à mort, puis guillotiné le 13 mai 1858.

Le gouvernement français feint alors de croire à l'organisation d'une vaste conspiration contre le régime et utilise ce prétexte pour mener des actions répressives contre l'opposition républicaine sur l'ensemble du territoire français. Les propos d'Espinasse, alors ministre de l'Intérieur et de la Sûreté Générale, dans sa circulaire du 23 février ${ }^{6}$ illustrent bien cet état de fait : " les démagogues qui se tenaient prêts à tenter un mouvement insurrectionnel si l'attentat du 14 janvier eût réussi veulent saisir l'occasion du jugement d'Orsini et de ses complices, pour faire une tentative de désordre. Le devoir du Gouvernement est de déjouer leurs projets ». Pour le gouvernement, il ne peut dépendre de quelques hommes de mettre en question l'autorité la plus légitime, les règles les plus respectées, les éléments les plus éclatants de l'ordre et du repos publics. Pour les mariannistes angevins, comme pour les autres opposants démocrates, cet attentat entraîne un raidissement de la politique intérieure qui se traduit par la diminution importante de l'octroi de grâces et un renforcement de la surveillance des milieux républicains et ouvriers. Espinasse demande alors au préfet de Maine-et-Loire de dresser des états nominatifs comprenant d'une part les individus, alors libérés ou graciés, qui ont été soit condamnés, soit internés, expulsés ou transportés par mesure de sûreté générale, à l'occasion des événements de mai et juin 1848, de juin 1849 et de décembre 1851, et d'autre part les individus condamnés pour appartenance à des sociétés

5. Sur l'attentat d'Orsini et ses conséquences générales sur la politique française, voir : GIRARD, Louis, Napoléon III, Paris, Librairie Arthème Fayard, 1986, p. 271-292.

6. Circulaire en date du 23 février 1858 du ministre de l'Intérieur adressée aux préfets français, Arch. dép. de Maine-et-Loire, 28 M 19, p. 2. 
secrètes, dont la conduite ne présente pas toutes les garanties et dont la présence dans le département offre des dangers pour l'ordre public. Un second tableau doit aussi être dressé, tableau où doivent être consignés les individus non-inscrits sur le premier tableau, mais devant tout de même être attentivement surveillés dans la mesure où ils pourraient prendre une part active à un mouvement insurrectionnel. Dans le même temps, le gouvernement et l'Empereur saisissent le corps législatif de l'examen d'un projet de loi ayant pour objet des mesures de sûreté générale et en particulier diverses dispositions spécialement applicables aux individus compromis dans les événements compris entre 1848 et $1851^{7}$. L'autorité judiciaire aide de façon active la police dans sa recherche d'individus suspects. En janvier, le Garde des Sceaux demande au procureur général d'Angers de " soumettre à un examen sérieux et ferme les actes et les paroles des hommes que leurs antécédents et leurs opinions hostiles signaleraient à de légitimes soupçons et de faire requérir des informations contre ceux qui paraîtraient avoir enfreint la loi pénale ${ }^{8}$ ».

\section{Les mariannistes "libérés ": entre étroite surveillance et répression}

Les nombreux états nominatifs envoyés à la préfecture par les bureaux de police locaux ${ }^{9}$ témoignent de l'ampleur de la surveillance policière : très vite, ce sont près de 600 individus qui figurent sur ces états ${ }^{10}$. Les communes d'Angers, des Ponts-de-Cé et bien sûr de Trélazé sont particulièrement surveillées, les autres arrondissements du Maine-et-Loire n'étant cependant pas en reste. La manière dont les mariannistes alors libérés ou les autres républicains et démocrates sont définis par les autorités de police est très révélatrice des inquiétudes et du climat d'alors. R. Bazille de Trélazé est décrit d'un " caractère sournois, méchant, toujours mécontent. [II] ne semble pas avoir renoncé à ses idées démocratiques [et] ne fréquente que des mariannistes". F. Manceau a, quant à lui, des " opinions exaltées, [un] caractère violent et dangereux [et est le] Beau-frère d'Attibert ${ }^{11}$ ", le meneur de l'insurrection de La Marianne en 1855 dont la femme a été emprisonnée en février 1858 pen-

7. Circulaire en date du 17 février 1858 du ministre de l'Intérieur adressée aux préfets français, Arch. dép. de Maine-et-Loire, 28 M 28.

8. Circulaire du ministre de la Justice au procureur général Métivier en date du 19 janvier 1858, Arch. dép. de Maine-et-Loire, 29 M 33.

9. Arch. dép. de Maine-et-Loire, 28 M 19, 28 M 20, 28 M 26, 28 M 28, et 28 M 30.

10. Dans Simon François, La Marianne, société secrète au pays d'Anjou. Essai d'éducation civique par l'histoire locale, Angers, Imprimerie angevine, 1939, p. 194-516, figurent plusieurs listes d'individus surveillés entre 1858 et 1861 . Il détaille à la page 515 la répartition des républicains socialistes ou socialistes démocrates surveillés dans chaque arrondissement du département : arrondissement d'Angers, 497 républicains socialistes ; arrondissement de Cholet, 44 socialistes démocrates; arrondissement de Saumur, 48 républicains socialistes ; arrondissement de Baugé, 27 républicains socialistes; arrondissement de Segré, 11 républicains socialistes.

11. Arch. dép. de Maine-et-Loire, 28 M 30. 
dant trois semaines en raison de la correspondance qu'elle entretient avec lui ${ }^{12}$. F. Chotard est "signalé comme très violent, comme très dangereux et comme exerçant une très pernicieuse influence dans la commune de Clefs dont la population a été gâtée par les sociétés secrètes ${ }^{13}$ ". J. Buteau, des Ponts-de-Cé est présenté comme un " citoyen de barricade, prêt à tous les événements pourvu qu'ils tendent à renverser l'État ${ }^{14}$ ". Dernier exemple, montrant que la surveillance n'oublie aucune contrée du Maine-et-Loire, U. Dagot est signalé comme un « démagogue incorrigible exerçant une très pernicieuse influence à Montreuil-Bellay ${ }^{15}$ ".

La circulaire du 23 février 1858 du ministre de l'Intérieur sollicite alors le préfet pour faire arrêter les dix hommes les plus dangereux du département. Ces individus doivent être choisis parmi ceux qui, condamnés depuis 1848, ont été libérés ou graciés, et ont cependant persisté dans "leurs coupables manœuvres". Les hommes ainsi arrêtés doivent être immédiatement enfermés dans la maison d'arrêt de l'arrondissement auquel ils appartiennent. Dans le Maine-et-Loire, dix républicains sont alors proposés pour être arrêtés. Il ne s'agit bien sûr pas des mariannistes ayant pris la tête de l'insurrection d'août 1855, ces derniers étant toujours en prison, au bagne de Guyane ou en exil ${ }^{16}$. Les dix hommes concernés demeurent en fait aux yeux des autorités les républicains libres les plus influents du département. Parmi eux, figure F. Taupin qui n'est plus domicilié dans le département, mais en Loire-Inférieure, à Nantes. Le préfet de Maine-et-Loire propose alors à son homologue du département voisin de le faire surveiller ${ }^{17}$. Les dix républicains que le préfet décide de faire arrêter sont finalement L. Bellanger, J. Butteau, F. Chotard, U. Dagot, F. Desportes, A. Dorgigné, F. Goré, P. Martin, N. Mauret, et J. Panneau ${ }^{18}$. Neuf d'entre eux sont arrêtés, puis entendus par les autorités, U. Dagot étant en fuite. Les interrogatoires ont, pour chacun d'eux, des issues très différentes. Pour les autorités, L. Bellanger, J. Butteau, F. Desportes et F. Goré " n'apparaissent pas dignes d'une quelconque clémence ". Ainsi, le préfet propose au ministre de l'Intérieur d'envoyer F. Desportes et F. Goré en Guyane avec U. Dagot, et d'interner en Algérie L. Bellanger et J. Butteau, ainsi que " pour l'exemple " N. Mauret et F. Chotard. De leurs côtés, A. Dorgigné, P. Martin et J. Panneau sont eux proposés pour une remise en liberté " sans que cela ne pose de réels dangers pour l'ordre public ".

12. Lettre non datée de François Attibert à son épouse, Arch. dép.de Maine-et-Loire, 29 M 33. Attibert qui s'est évadé du bagne de Cayenne en 1856 est alors à Londres.

13. Arch. dép. de Maine-et-Loire, 28 M 19.

14. Arch. dép. de Maine-et-Loire, 28 M 26.

15. Arch. dép. de Maine-et-Loire, 28 M 19.

16. Lettre du préfet de Maine-et-Loire au ministre de l'intérieur en date du 24 février 1858, Arch. dép. de Maine-et-Loire, 28 M 19, p. 8.

17. Lettre du préfet de Maine-et-Loire à son homologue de Loire-Inférieure en date du 27 février 1858, Arch. dép. de Maine-et-Loire, 28 M 19, p. 7.

18. État nominatif des individus proposés pour être arrêtés et lettre du 6 mars 1858 du préfet de Maine-et-Loire au ministre de l'Intérieur et de la sûreté générale, Arch. dép. de Maine-et-Loire, 28 M 19, p. 8-9. 
On franchit un nouveau palier dans la répression avec le vote de la loi de Sûreté Générale du 27 février 1858 dont l'article 7 prévoit en effet d'interner dans un département de l'Empire ou en Algérie, ou d'expulser du territoire, " tout individu qui a été, soit condamné, soit interné, expulsé ou transporté, par mesure de sûreté générale, à l'occasion des événements de mai et juin 1848 , de juin 1849, ou de décembre 1851, et que des faits graves signaleraient de nouveau comme dangereux pour la sûreté publique ». Le cadre juridique d'une telle mesure étant très vaste, de nombreux mariannistes du Maine-et-Loire condamnés en 1855 et 1856 sont concernés. Outre son caractère arbitraire, cette loi s'apparente ainsi à une " loi des suspects", même si comme le souligne l'article 10, la mesure de sûreté générale n'est prise par le ministre de l'Intérieur que « sur l'avis du préfet du département, du général qui y commande et du procureur général ", c'est à dire sur l'avis des commissions mixtes (de 1852). Siégeant à la préfecture où doivent être rassemblés les documents de diverses provenances, toutes les pièces de procédure et les informations sur les individus jugés dangereux pour l'ordre public, elles ne prononcent pas de réels jugements mais prennent à l'égard de chaque inculpé une décision, signée par ses trois membres, qu'elles soumettent au gouvernement de l'Empereur. Il revient ensuite légalement au gouvernement de condamner l'inculpé, par l'intermédiaire d'un arrêté conforme à la décision de la commission départementale ${ }^{19}$. Dans le département de Maine-et-Loire, comme dans tout le pays ${ }^{20}$, la surveillance policière permet de recueillir de nombreux renseignements à propos d'individus jugés dangereux, qui sont ensuite jugés par la commission mixte.

Aussi vaste que soit l'arsenal répressif mis en place, le passé de certains condamnés, notamment de certains du Maine-et-Loire, ne correspond pas aux cadres juridiques définis par la loi de sûreté générale. En effet, l'affiliation à une société secrète n'y est pas clairement établie comme un des délits punissables. Devant ce vide juridique, les autorités se réfèrent alors aux articles 1 et 2 du décret du 8 décembre 1851, articles qui avaient déjà été utilisés en 1855 pour transporter en Guyane les quatre mariannistes angevins, A. Arridas, R. Cheigneau, C. Gabaston et V. Riotteau, alors qu'ils

19. Dans les faits, la commission mène deux types d'opérations : l'une de forme judiciaire, à savoir statuer sur le sort d'individus compromis dans des événements depuis 1848 et qualifiés d'inculpés comme s'il s'agissait d'un jugement; une autre de type purement politique, débarrasser la société française de personnes considérées dangereuses pour l'ordre public et social. Sa composition et la nature des opérations qu'elle est chargée de mettre en œuvre font donc d'elle une juridiction administrative. La décision des trois membres de la commission est prise en secret, sur des pièces écrites ou des renseignements privés, sans que le détenu soit entendu, et sans que ce dernier ait un quelconque droit à se défendre ou à se rétracter de ses " erreurs " passées. Il ne s'agit en fait que d'une mesure de sûreté générale que le pouvoir exécutif reste maître de modifier. Pour chaque délit ou crime, la loi de Sûreté Générale prévoit une peine précise, l'accusé ou le prévenu concerné pouvant cumuler les fautes, donc les peines.

20. Sur tout le territoire français, ce sont environ 400 individus suspectés de travailler à un complot républicain et socialiste d'envergure nationale qui sont condamnés par ces commissions mixtes. 
n'avaient été condamnés qu'à de courtes peines d'emprisonnement pour " affiliation à la Marianne ". Au cours du printemps et de l'été 1858, une vingtaine de républicains du Maine-et-Loire, mariannistes ou non, sont ainsi condamnés par décret ministériel à l'internement en Algérie (L. Bellanger, M. Blin, J. Buteau, F. Chotard, F. Coulbault, F. Desportes, J.-F. Domont, J. Dorgigné, F. Goré ${ }^{21}$, P. Grosset, R. Guillot, V.-E. Huard, P. Martin, N. Mauret) ou en Guyane ${ }^{22}$ (L. Bottereau et H. Dudouet) ${ }^{23}$.

Ainsi, à l'échelle nationale, la politique de répression menée par Espinasse atteint des sommets avec la loi de sûreté générale le 27 février 1858. Jusque-là, il pouvait paraître utile aux autorités, selon leur volonté de régulation politique et sociale, de stigmatiser les opposants républicains en leur offrant la clémence impériale, en les soumettant ainsi au régime et à l'autorité de l'Empereur. Développer, dans les masses populaires, un sentiment de gratitude à l'égard de celui qui pardonne était une politique à laquelle nombre de responsables administratifs et judiciaires adhéraient. Mais le vote de cette loi et l'ampleur de la répression révèlent aussi les craintes et la fragilité du pouvoir. Se rendant hostile une grande partie des masses populaires, Napoléon III comprend très vite qu'une nouvelle orientation au régime est un impératif vital pour le régime.

« Napoléon aim[ant] à penser que les victoires [...] opér[ent] la réconciliation des partis ${ }^{24}$ " et conscient de ne pas être en mesure de prendre des initiatives à l'intérieur du pays, il décide alors d'agir à l'extérieur, en particulier en Italie où il souhaite participer militairement à l'unification du pays et défendre le principe révolutionnaire des nationalités cher aux républicains.

\section{Soutien impérial aux nationalistes italiens et répercussions dans la politique intérieure de Napoléon III}

Après des pourparlers secrets, un traité est signé avec le Piémont le10 décembre 1858, ce qui ouvre la voie à une intervention militaire en Italie du nord. Entre mai et juillet 1859, l'Empereur honore ses engagements et lutte contre l'armée autrichienne. Les deux victoires de Magenta (le 4 juin) et de Solférino (24 juin) lui offrent un prestige important. L'armistice signé le 11 juillet à Villafranca, sans que tous les objectifs initiaux aient été atteints (la Vénétie restant par exemple sous domination autrichienne, ce qui déplaît fortement aux Italiens) témoigne surtout que l'Empereur est pressé d'en finir, la Prusse se faisant de plus en plus menaçante, l'opinion française n'étant alors pas prête à une guerre longue. Quoi qu'il en soit,

21. F. Goré s'est alors enfuit en Amérique.

22. Arch. dép. de Maine-et-Loire, 28 M 18, 28 M 20 et 28 M 26.

23. P.-F. Dubois, P. Millault, J. Panneau, A. Pauvert, R. Piault et J. Rosée sont eux aussi condamnés à l'internement en Algérie ou à Cayenne, mais aucune archive consultée n'indique où ils y ont été conduits pour subir leurs peines.

24. Sur ce point, voir GIRARD, Louis, Napoléon III, op. cit., p. 292. 
l'Empereur a combattu l'Autriche, une des puissances européennes les plus conservatrices, en défendant le principe des nationalités. Mais, en s'engageant aux côtés des nationalistes italiens, il est allé aussi contre les intérêts du pape dont le pouvoir temporel est en effet menacé par l'unification italienne. L'Italie réunifiée, il est bien délicat d'imaginer une autre capitale que Rome qui est aussi celle des États pontificaux. Or, de nombreux cléricaux, en France comme à l'étranger, pensent que le pape ne peut être complètement libre et indépendant s'il ne dispose pas d'un État et d'une capitale symbolique. En 1859, il perd la plus grande partie des États pontificaux, ne conservant que Rome et le Latium. Les cléricaux français, qui constituaient jusque là une des bases sociales du régime impérial, sont furieux. Cette base sociale vitale pour le régime, Napoléon III doit la modifier. Comme le souligne Stéphane Gacon, « Napoléon III, qui observe avec inquiétude l'érosion d'une partie de ses appuis traditionnels et la persistance d'un sentiment républicain, aurait choisi de jouer la carte populaire, quitte à s'aliéner les premiers pour désamorcer la menace de la montée en puissance du second ${ }^{25}$ ". Signe de l'orientation politique plus "libérale " du régime, il décrète le 16 août 1859 l'amnistie pour les condamnés politiques depuis 1848.

\section{Le décret impérial d'amnistie du 16 août 1859 pour les condamnés politiques depuis 1848}

Théoriquement, une amnistie est une décision du pouvoir législatif (sensé être le corps représentatif de la nation) qui efface un fait punissable, arrête les poursuites et anéantit les condamnations. En cela, elle diffère de la grâce, accordée par le chef de l'État qui ne fait que supprimer l'exécution de la peine en laissant subsister les effets de la condamnation. Signe de négation et de contournement d'une des rares fonctions théoriques de l'assemblée législative, le décret impérial d'amnistie du 16 août 1859 marque l'hégémonie de la personne impériale dans le domaine de la justice et plus particulièrement dans celui du pardon judiciaire. Le but de cette décision étant d'attacher le prestige de ce pardon à la personne impériale, les amnistiés devront reconnaître en elle la seule autorité pouvant pardonner, et finalement la seule méritant une quelconque gratitude. Il s'apparente donc dans les faits à une grâce amnistiante collective ${ }^{26}$.

25. GACON, Stéphane, " 1859 et 1879 : deux amnisties ", in CARON, Jean-Claude, ChAUvAUD, Frédéric, FuREIX, Emmanuel et Luc, Jean-Noël, Entre violence et conciliation. La résolution des conflits sociopolitiques en Europe au XIX siècle, Rennes, PUR, 2008, p. 352.

26. Sur ce point, voir GACON, Stéphane, L'Amnistie. De la Commune à la guerre d'Algérie, Paris, Éditions du Seuil, 2002, 428 p. Plus largement, à propos des formes de pardon judiciaire, voir ZEmon-DAVIs, Nathalie, Pour sauver sa vie. Les récits de pardon au XVte siècle, Paris, Seuil, 1988, 283 p. et COLTEL, Antony, "Le pardon ou l'oubli? La réhabilitation judiciaire sous la III" République : le cas d'Angers ", Crime, Histoire et Sociétés, 11, 2, 2008, p. 89-106. 


\section{Revenir de prison et du bagne}

Pour ce qui est des mariannistes, la plupart de ceux condamnés en 1855 et 1856 ont déjà été libérés au moment où l'amnistie est décrétée. Ayant obtenu une grâce, pour quelques-uns, ou ayant effectué la totalité de leur peine, nombreux sont ceux qui sont désormais libres, aux côtés de leurs familles. Seuls les " incorrigibles " et ceux à nouveau condamnés depuis leur libération sont encore en prison, en détention en Algérie, au bagne de Guyane ou en exil. Si tous les condamnés de 1855 et 1856 sont concernés par ce décret, les conséquences réelles d'une telle mesure ne sont pas ressenties de manière uniforme par tous. Obtenir sa libération de prison, du bagne et/ou le droit de revenir en France après avoir passé quelques années d'exil en Angleterre est évidemment d'une autre importance que de voir simplement son passé de " délinquant " ou de " criminel " ne plus être considéré comme tel alors que l'on est déjà chez soi.

Selon les cas, les mariannistes libérés, suite à l'amnistie du 16 août 1859, reviennent en Anjou entre août 1859 et janvier 1860. Ceux revenant le plus tôt sont bien sûr les hommes jusqu'alors prisonniers dans les maisons centrales métropolitaines de Beaulieu (à Caen) et de Limoges. J.-C. Béziau, U. Bridier, A. Boilême, P. Cordier, J. Girard, A. Goré, L. Guy, H. Janvier, L. Lemeunier, J. Richard, F. Trideau et U. Ubarain reviennent en Anjou dès la fin du mois d'août $1859^{27}$.

Les déportés amnistiés de l'île du Diable arrivent en Anjou courant janvier 1860, après avoir été débarqués, pour une partie, (sauf H. Briand ${ }^{28}$ ) le 7 janvier à Toulon par le Cérès en compagnie d'une trentaine d'autres amnistiés de toute la France ${ }^{29}$. Les évadés du bagne reviennent quant à eux au cours du dernier trimestre 1859.

27. Dans Simon, François, La Marianne, société secrète..., op cit., p. 185-187, figure un état nominatif de 46 mariannistes libérés à la suite de l'amnistie du 16 août 1859, et pour certains la date de leur retour en Anjou. Il s'agit d'individus condamnés en 1855 et 1856 à l'emprisonnement et à la déportation. L'étude de Denise Devos, La Troisième République et la mémoire du coup d'Etat de Louis Napoléon Bonaparte. La loi de réparation nationale du 30 juillet 1881, Paris, Archives Nationales, 1992, p. 320-322, offre pour chaque département une liste des individus ayant réclamé à l'État républicain des années 1880 des réparations pour les peines auxquelles ils ont été condamnés sous l'Empire. Y figurent les dates de décès des victimes du régime impérial, ce qui permet de dire que certains noms donnés par François Simon sont ceux d'individus décédés au moment de l'amnistie. Quant au témoignage de François ATTIBERT, Quatre ans à Cayenne, Bruxelles, Vve Verteneuil, 1859, in-12, il offre des informations étonnantes sur la vie et la mort des Mariannistes déportés. Les récits de la mort de certains de ses compagnons, comme celle de G. Lapierre, permettent là encore de préciser l'état nominatif de François Simon.

28. La date et les moyens utilisés par H. Briand pour son retour ne sont mentionnés ni dans les archives, ni dans les sources qui ont été consultées.

29. Pour les mariannistes, il s'agit de L. Auray, H. Briand, J.-B. Chauvin, R. Deshayes, L. Fouin, E. Frouin., N.F. Gaignard., F. Manceau, F. Thiberge et J.-P. Vivant. D'après C. Aubert, F. Guérin, évadé de Cayenne avec Attibert, demeure aux États-Unis avec sa famille, dans l'État de New York, à Rockland County. Voir AUBERT, Christophe, La Répression des manifestations séditieuses de l'opposition politique en Maine-et-Loire au XIXe siècle (1814-1870), thèse d'État, UFR de droit de Rennes I, 1995, T. II, p. 502. Pour les dates d'arrivée de 
Ceux condamnés à la transportation lors de la répression de 1858 sont aussi compris dans le décret impérial d'amnistie du 16 août 1859 . Retrouvant alors leur liberté, un certain nombre d'entre eux, la plupart même, reviennent en Anjou ${ }^{30}$. Certains quittent donc l'Algérie ou la Guyane où ils ont passé quelques semaines, voire quelques mois. Condamné à la transportation outre-atlantique le 20 juin 1859, L. Bottereau est de retour à Toulon le 31 août suivant, date à laquelle lui est délivré un passeport pour se rendre à Trélazé ${ }^{31}$. Pour certains, la situation semble plus confuse. La femme et la fille de Pierre Grosset, de Trélazé, se rendent en Algérie durant le dernier trimestre de $1858^{32}$. Est-ce pour passer quelque temps en compagnie du transporté dont le retour aurait été retardé pour une raison inconnue, ou pour s'y installer, avec lui, de manière définitive? Quoi qu'il en soit, l'amnistie du 16 août 1859 permet à tous les mariannistes encore prisonniers ou déportés de rentrer en Anjou. Ces hommes retrouvent donc leurs familles, après plus de quatre ans de séparation pour certains.

Dès leur arrivée en métropole, les libérés sont l'objet de toutes les attentions des administrations impériales, locales ou nationales. Lorsque le ministre de l'Intérieur, le Duc de Padoue, apprend l'arrivée à Toulon, le 7 janvier 1860, des mariannistes angevins, il demande au préfet de Maine-etLoire, Léopold Bourdon de Rouvre ${ }^{33}$, de les faire surveiller « d'une manière ostensible " et de le tenir informé " de l'effet produit par leur rentrée en France, ainsi que l'attitude qu'ils auront eux-mêmes gardée ${ }^{34}$ ". La surveillance de la haute police est bien entendu maintenue. La plus petite possibilité de trouble public obsède les pouvoirs politiques à tous les niveaux. Toutes les rumeurs, tous les faits inhabituels sont observés et mis en lumière. Comme l'a montré François Ploux pour tout le XIX ${ }^{\mathrm{e}}$ siècle, l'administration préfectorale et judiciaire est complètement obsédée par la rumeur et la recherche de ses origines. "Tous considèrent à priori la rumeur comme le résultat d'une manipulation de l'opinion à des fins sub-

L. Fouin et d'E. Frouin, voir la lettre du commissaire central d'Angers au préfet en date du 31 janvier 1860, Arch. dép. de Maine-et-Loire, 28 M 20, p. 13. Pour J.-P. Vivant, voir la lettre du commissaire central d'Angers au préfet de Maine-et-Loire du 27 janvier 1860, Arch. dép. de Maine-et-Loire, 28 M 20, p. 12.

30 . Nous ne disposons dans les archives que de quelques exemples, ce qui ne nous permettra pas de détailler la date et les conditions du retour de chacun des condamnés concernés et évoqués plus haut.

31. Bottereau, comme tous les libérés, est muni à sa sortie du bagne (ou de prison) d'un passeport sur lequel figurent son état-civil, son signalement physique, le lieu où il a déclaré vouloir fixer sa résidence après sa libération ainsi que de nombreux autres renseignements.

32. Liste départementale des émigrés du Maine-et-Loire vers l'Algérie (1855-1877), Arch. dép. de Maine-et-Loire, 6 M 74, liasse 5.

33. Il remplace Paul L.M.A. Vallon à cette fonction le 13 novembre 1857. À propos des préfets du Maine-et-Loire, voir MARAIS, Jean-Luc, Les Préfets de Maine-et-Loire, Rennes, PUR., 2000, 305 p.

34. Lettres du ministre de l'Intérieur au préfet de Maine-et-Loire de janvier 1860, Arch. dép. de Maine-et-Loire, 28 M 23, p. 1 et 2. 
versives $^{35}$. "Selon une lettre formatée, le commissaire central de police d'Angers informe le préfet des arrivées à Angers des anciens condamnés mariannistes ${ }^{36}$. Les renseignements fournis par le commissaire de police spécial de Trélazé Bugnon au préfet de Maine-et-Loire à propos des visites chez F. Attibert témoignent aussi de cette attention toute particulière : " aussitôt son arrivé [sic], Manceau s'est rendu chez Attibert son beaufrère, l'entretien a été long (Deux heures) dit-on, Attibert, avait reçu une lettre de Londres le 9 ce qui lui en fait deux de plus depuis 12 jours, rien ne transpire sur le contenu de ses lettres, il est très défiant et se tient sur ses gardes. Le Pays est très tranquille, les esprits sont calmes, le travail sur les carrières marche assez bien ${ }^{37}$ ". Si la surveillance des mariannistes revenus en 1859-60 vient s'ajouter à celle visant ceux libérés plus tôt, elle constitue une réalité avérée dès leur arrivée sur le sol français métropolitain et se renforce les semaines et les mois suivants.

\section{Le maintien de la surveillance et de la répression impériales après l'amnistie du 16 août 1859}

Pour les autorités, les révoltés qui reviennent en 1859-1860 constituent donc une menace dans la mesure où ils sont susceptibles de cristalliser autour d'eux les mécontentements d'une partie de la population ouvrière et à terme de réorganiser une opposition dangereuse pour l'ordre napoléonien. S'ils doivent compter avec le manque de fermeté de la conscience politique ouvrière en ce milieu de $\mathrm{XIX}^{\mathrm{e}}$ siècle, ils jouissent tout de même auprès de nombreux carriers d'une aura exceptionnelle. En prison, au bagne ou en exil (en Angleterre), certains ont été en contact avec les plus grands résistants au despotisme impérial. Quelques-uns, comme Attibert, se sont échappés de Guyane avec un courage reconnu par tous. Les inquiétudes des autorités sont donc légitimement vives et la surveillance active. On rapporte ainsi que, le 24 mars 1860, vers 19 heures, Attibert, Bottereau, Maillard, Hamard, Bellanger et d'autres anciens mariannistes (en tout une dizaine) se sont réunis dans le cabaret des époux Gourdon à La Pyramide (à Trélazé) " où ils se sont occupés de politique ". Attibert y aurait dit qu'Orsini, après avoir manqué son coup, lui a écrit une lettre pour exposer les malheurs qui guettaient les républicains français pour l'année 1858. Il ajoutait alors avoir reçu une autre lettre sans dire ce qu'elle contenait ni d'où elle venait. Un de ses assistants aurait alors ajouté : "Si cela recommence on saura mieux s'y prendre pour réussir. "

35. Ploux François, " Des bruits alarmants ", Les Cahiers de Médiologie, 13 - La scène terroriste, Septembre 2002, p. 91.

36. Lettres du commissaire central de police d'Angers au préfet de Maine-et-Loire datées des 27 et 31 janvier 1860, Arch. dép. de Maine-et-Loire, 28 M 20, p. 12 et 13.

37. Lettre du commissaire de police spécial en poste à Trélazé au préfet de Maine-etLoire en date du 12 janvier 1860, Arch. dép. de Maine-et-Loire, 28 M 20, p. 11. L'orthographe est respectée. 
Pour les autorités, la surveillance policière s'accompagne du souci de réprimer et de se débarrasser des " agitateurs ". Le 2 avril 1860, à Trélazé, Attibert, Bottereau, Deshaies, Girouard et Hamard sont arrêtés pour injures à des contremaîtres, pour avoir fait pression sur d'autres ouvriers afin qu'ils fassent grève si les salaires n'étaient pas augmentés et pour affiliation à une société secrète. Le ministre de la Justice en appelle même au procureur général Métivier : "J'appelle votre surveillance la plus attentive sur la prévention de coalition comme sur celle d'affiliation à une société secrète et vous prie de provoquer une sévère répression à l'égard de tous les inculpés, notamment à l'égard de ces hommes incorrigibles qui se montrent si peu dignes de la clémence dont ils ont été l'objet ${ }^{38}$. " Cette arrestation a en fait pour but de " les intimider et de les rappeler à la raison ${ }^{39}$ " et une ordonnance de non-lieu est rendue le 23 avril suivant ${ }^{40}$. Le mois suivant, Attibert et Bottereau, ne trouvant pas de travail dans les carrières de Trélazé, émettent l'idée de partir en chercher en Espagne où existent aussi des exploitations minières d'ardoise. Les autorités sautent sur l'occasion pour favoriser leurs départs. Le ministre de l'Intérieur affirme au préfet de Maine-et-Loire que " les carrières seront débarrassées de ces deux anciens affiliés de la Marianne, qui entretiennent constamment l'agitation et l'esprit de révolte " et ordonne ensuite : "Payez les voyages d'Attibert sur les fonds secrets, sans qu'il le sache, et obtenez du ministre de l'Algérie le passage gratuit pour Bottereau ${ }^{41}$. " Mais ces tentatives échouent successivement et les mariannistes influents continuent de travailler, avec peu de succès d'ailleurs, à la reconstitution d'une opposition républicaine locale au régime impérial.

Si des signes attestent de la survie et du développement d'organisations républicaines au niveau national, comme la société " les Faucheurs ", l'opposition républicaine en Anjou s'essouffle en ce début de décennie 1860 et la situation politique est globalement calme.

L'arrivée au pouvoir municipal à Trélazé en 1861 de J.-B. Fourcault qui saura très vite se faire apprécier de ses administrés, notamment en croisant le fer avec la commission des Ardoisières et en développant l'école pour les enfants d'ouvriers, ainsi que la disparition tragique de François Attibert en 1866, renforcent un peu plus cette situation défavorable à l'opposition républicaine et socialiste en Anjou. Attibert s'éteint en effet le 7 août 1866 des suites de blessures reçues le dimanche précédent sur la route des Ponts-de-Cé à La Pyramide, où il a été renversé par une voiture de boucher. L'enterrement a lieu deux jours plus tard à Trélazé à cinq heures

38. Lettre du ministre de la Justice au préfet de Maine-et-Loire en date du 4 avril 1860, Arch. dép. de Maine-et-Loire, 29 M 33.

39. Passage d'une lettre du procureur général cité dans Simon François, La Marianne, société secrète..., op. cit., p. 217.

40. Lettre du procureur impérial Chevalier au procureur général Métivier du 23 avril 1860, Arch. dép. de Maine-et-Loire, 29 M 33.

41. Passage d'une lettre du ministre de l'Intérieur au préfet de Maine-et-Loire cité dans Simon François, op. cit., p. 189. 
du soir. Sont présents quelques lieutenants de l'ancien général de la révolte, quelques membres de la société de secours mutuels des Justices dont il faisait partie et quelques parents du défunt. Pour J.-B. Fourcault, présent à la sépulture, cette cérémonie se passe globalement sans manifestation, sans cri. Chacun raconte son évasion de Cayenne, tandis que quelques rares propos malveillants à son égard viennent troubler le recueillement des proches d'Attibert : "S'il était mort il y a quinze ans, nous n'aurions pas commis l'horrible forfait de $1855^{42}$. " Quoi qu'il en soit, pour beaucoup, il était " un homme énergique " et " une des plus grandes figures de la Marianne et du Peuple angevin ${ }^{43}$ ".

La décision de Napoléon III d'amnistier les condamnés politiques de l'Empire est donc à comprendre dans un contexte politique national et international particulier. Si elle répond «à des formes constitutionnelles révélatrices de la nature du régime ${ }^{44}$ ", elle demeure un instrument remettant en cause la chose jugée, le jugement et l'autorité qui juge. La façon dont l'Empereur contourne une des attributions du pouvoir législatif au moment de l'amnistie en offre un exemple flagrant, faisant apparaître ce type de clémence impériale comme une grâce collective amnistiante.

Cette amnistie "ferme aussi l'épisode aigu de crise civile ». Mettant fin aux affrontements publics de la première décennie du règne de Napoléon III, " elle contribue à pacifier, au moins pour un temps le débat public ${ }^{45}$ " national ou local.

Cette amnistie marque la fin de l'épisode " carcéral " de la Marianne et permet à ceux qui n'ont pas obtenu de grâce ou qui se sont évadés de Cayenne de recouvrer leur liberté et de revenir en Anjou. Or, ces " libérés de 1859 " doivent très vite composer avec des autorités qui ne les ont pas oubliés tant ils demeurent dangereux pour l'ordre social impérial et gardent la marque de leurs actes passés. L'étude de la correspondance des administrations locales ou nationales révèle leurs vives inquiétudes quant à l'impact, sur la population, du retour de ces bagnards et de ces prisonniers. La surveillance et la menace d'une forte répression incitent donc à la prudence tout en retardant la réorganisation politique des masses ouvrières angevines.

Ce n'est véritablement qu'avec la loi sur les indemnités à accorder aux victimes du coup d'État et à la loi de Sûreté Générale votée le 30 juillet $1881^{46}$

42. Lettre de J.-B. Fourcault au préfet de Maine-et-Loire datée du 9 septembre 1866, Arch. dép. de Maine-et-Loire, 28 M 17, p. 4. La lettre a été écrite le lendemain de l'enterrement de F. Attibert. Or, elle est datée du 9 septembre 1866. Il s'agit sans doute d'une erreur, Attibert étant décédé le 7 août. Cette lettre a donc sans doute été écrite le 9 août et non le 9 septembre, F. Attibert ayant alors été enterré le 8 août.

43. SimON, François, La Marianne, société secrète..., op. cit., p. 190.

44. GACON, Stéphane, " 1859 et 1879 : deux amnisties ", op. cit., p. 351.

45. Ibidem, p. 353.

46. Seules quelques familles de mariannistes en bénéficieront malgré de nombreux requérants. Les familles obtenant réparation sont celles d' A. Arridas, F. Attibert, M. Blin, 
que l'affaire de La Marianne se termine : la République alors naissante s'attache à réparer un douloureux passé politique pour s'ancrer véritablement auprès des populations concernées par la répression impériale, en faisant de la résistance au despotisme napoléonien un combat pour le droit.

Si elle correspond pour l'historien à « une pratique anti-historique étonnante parce qu'elle veut enfermer dans l'oubli tout un pan du passé ${ }^{47}$ ", l'amnistie n'entraîne pas pour autant l'amnésie. Le souvenir, la mémoire des luttes contribuent " à structurer les futurs antagonismes sociaux et politiques $^{48}$ ". À Trélazé en particulier, les luttes politiques et sociales ne disparaissent bien évidemment pas avec la Marianne. Fortement teintées d'anarcho-syndicalisme, elles reprennent plus vivement au tournant du siècle, notamment sous l'influence de Ludovic Ménard, une autre, voire la figure emblématique du syndicalisme ouvrier et ardoisier de la ville. "Apparten[ant] à la légende du prolétariat trélazéen ${ }^{49}$ ", la Marianne constitue encore aujourd'hui pour certains ouvriers angevins, en particulier les ardoisiers de Trélazé, ainsi que pour une partie de la classe politique locale, un moment historique fort, symbole de la lutte des humbles contre l'oppression bourgeoise.

\footnotetext{
L. Bottereau, J. Butteau, F. Desportes, J. Dorgigné, P.-F. Dubois, F. Goré, F. Guérin et J. Panneau. Voir sur la loi de réparation nationale de 1881 : Devos, Denise, La Troisième République et la mémoire du coup d'Etat de Louis-Napoléon Bonaparte. La loi de réparation nationale du 30 juillet 1881, Paris, Archives Nationales, 1992 et DEvos, Denise, " La loi de réparation nationale du 30 juillet 1881 : source de l'histoire de la répression de l'insurrection de décembre 1851 ", Revue d'histoire du XIXe siècle, 1, 1985, [En ligne], mis en ligne le 28 octobre 2002. [http://rh19.revues.org/index3.html].

47. GACON, Stéphane, L'Amnistie..., op. cit., p. 13.

48. GACON, Stéphane, " 1859 et 1879 : deux amnisties », op. cit., p. 353.

49. Tноме́, Jacques, Trélazé, cité des faiseurs d'ardoise (1608-1918), Angers, Ville de Trélazé, 1988, p. 176.
} 


\section{RÉSUMÉ}

En janvier 1860, après quatre longues années de bagne guyanais pour certains, les derniers mariannistes condamnés à la suite de l'insurrection angevine de 1855 reviennent en Anjou. La décision de Napoléon III d'amnistier les condamnés politiques de l'Empire le 16 août 1859 est à comprendre dans un contexte national et international complexe.

Si le décret d'amnistie traduit à la fois la nature, le fonctionnement, les hésitations et finalement les évolutions libérales du régime, il apparaît aussi comme un instrument de régulation politique et sociale dans les mains de la personne impériale.

L'amnistie ferme l'épisode " carcéral " de l'affaire de La Marianne mais ces " libérés de 1859 " doivent très vite composer avec des autorités impériales locales et nationales qui ne les ont pas oubliés tant ils demeurent à leurs yeux de potentiels dangers pour l'ordre impérial.

\section{ABSTRACT}

In January 1860, after 4 long years in the Guyana penal colony for some, the last "Mariannistes" who were condemned after the Angevine insurrection of 1855 came back to Anjou. Napoleon III's decision to amnesty the political convicts of the Empire on August 16th 1859 must be understood against a complex national and international background.

If the Amnesty Decree showed the nature and working, the hesitations and then the liberal evolutions of the regime, it also appeared as an instrument of political and social control in the hands of the Emperor.

The Amnesty put an end to the "La Marianne prison affair" but the "1859 freed ones" had to face local and national imperial authorities that hadn't forgotten them, as they remained a potential danger for imperial order. 